\title{
Do Engineered Nanoparticles Penetrate into Cells?
}

\section{Komal Garde and Shyam Aravamudhan*}

Department of Nanoengineering, Joint School of Nanoscience and Nanoengineering, North Carolina A\&T State University, USA

Understanding the mechanism of engineered nanoparticle (ENP) uptake by cells is important for various biomedical applications including biosensors, imaging, intracellular drug and gene delivery, and toxicity studies [1-3]. This editorial presents a short summary and perspective on the cellular uptake of gold nanoparticles and the mechanisms that govern them. Gold Nanoparticles (GNPs) have been proposed for use in practically all biomedical applications because of the ease in synthesis, chemical stability, and their unique optical and electrical properties [4]. But, spontaneous penetration of functionalized cationic GNPs have shown cell membrane disruption and cytotoxicity, thus limiting their utility [5]. However, recent literature has shown that GNPs (5nm diameter with "special" surface chemistries or arrangements) protected by an amphiphilic monolayer can nondisruptively penetrate the cell membrane to deliver drugs, nutrients or biosensors [6]. Even with endocytosis arrested, this penetration via an energy independent mechanism do not damage or rupture cell membrane [7]. The underlying mechanism of how GNPs can nondisruptively penetrate cell membranes has largely been unknown [8]. Recently, Van Lehn et al. [9] demonstrated that the penetration process consists of multiple steps - (a) first the anionic, striped GNPs will fuse with the cell membrane in a non-disruptive transmembrane configuration, (b) then the GNPs may translocate into the cell interiors. This process is dependent among others on particle size, monolayer composition, and ligand morphology.

GNP size is found to play a critical role in both the rate and extent of cell uptake. Chithrani et al. found that $50 \mathrm{~nm}$ transferrin-coated GNPs are taken up by mammalian cells at higher rates and extents compared to other sizes in the range of 10-100 nm [10]. Chithrani et al. [11] suggested "wrapping effect" based on free energy calculations and receptor diffusion kinetics as the basis for how fast and how many NPs are internalized in the cell. Various tools are available to image (using either light or electron microscopy) and quantify (elemental analysis) GNPs concentration inside the cell. Elemental detection techniques such as ICP coupled with Mass Spectroscopy (ICP-MS) is an excellent technique to analyze gold content both inside and outside (growth media) of the cells. In addition, because GNPs are electrondense, TEM techniques can be used to distinguish gold from other cellular components. Depending on the GNP size, type, cell receptors and cellular signaling cascades, various other pathways such as phagocytosis, micropinocytosis, and receptor-mediated endocytosis (RME) including caveolae-mediated, clathrin-mediated, and caveolae/ clathrin independent endocytosis have been proposed [12].

Many researchers have studied how different cells selectively respond to GNPs. For example, in one study, BHK21 and HepG2 cells showed no effect, while A549 cells underwent apoptosis due to accumulation of GNPs in periphery outside cell nucleus [13]. In another work, the mechanisms by which transferrin-coated GNPs entered three cell lines (STO, HeLa, and SNB19) varied [10]. Ultimately, to understand and predict the relationship between NP size and different cell line exocytosis, free energy mathematical models were developed [10].

As stated earlier, microscopic imaging can be used to study GNP internalization and accumulation in different parts inside the cell. It is important to consider cell internalization as a function of size, incubation time, temperature, and surface functional group [14]. Elemental analysis using ICP OES have shown abundant internalization and nuclear localization of gold complexes. This nanoparticle internalization is largely governed by the adsorbed proteins and their relative orientation on the curved nanoscale surface. Depending on chemical faces, receptors can mediate different pathways for cell entry. Negatively charged GNPs are shown to adsorb serum proteins and enter cells via a complex endocytic pathway resulting in higher toxicity and immunological response [15]. GNP internalization can also be manipulated by changing the surface chemistry, polyelectrolytes and surfactants of varying charge. TEM studies have shown GNPs coated with quaternary amines (CTAB and PDAD-MAC) exhibit higher uptake, compared to molecules containing primary amine (PAH) [16]. Cho et al. [17] observed that surface functionalized groups have a stronger influence on cell uptake than its shape. In addition, GNPs have exhibited particle size dependent organ distribution after intravenous administrationin rats. ICP-MS studies indicate that $10 \mathrm{~nm}$ GNPs are present in blood, liver, spleen, kidney, testis, thymus, heart, lung and brain, while 50-250 nm GNPs are detected only in blood,liver and spleen [18].

Although it seems that GNPs can be taken up by different types of cells, the evidence is disparate and the mechanisms of uptake are either not examined or in their infancy [9]. It also remains unclear whether GNPs taken up by cells exert a cytotoxic effect or not. Accurate and comprehensive physical and chemical characterization data becomes very important to describe both toxicity and the cellular uptake mechanism. Currently, a diverse assortment of nanomaterials and experimental conditions are available, with no reliable and reproducible methods, models and standardized engineered nanomaterials. In future, a more ordered and systematic approach (with computational and experimental methodology) is necessary to both assess the biological responses and to address fundamental mechanistic questions.

\section{Acknowledgement}

The authors acknowledge funding support from Semiconductor Research Corporation/ Nanomanufacturing Sciences, ESH (Task ID. 425.037) and SRC Engineering Research Center for Environmentally Benign Semiconductor Manufacturing at University of Arizona, Tucson.

\section{References}

1. Han G, Ghosh P, Rotello VM (2007) Functionalized gold nanoparticles for drug delivery. Nanomedicine 2: 113-123.

*Corresponding author: Shyam Aravamudhan, Department of Nanoengineering, Joint School of Nanoscience and Nanoengineering, North Carolina A\&T State University, 2907 E Lee Street, Greensboro, NC 27401, USA, Tel: (336) 285-2856; E-mail: saravamu@ncat.edu

Received October 15, 2013; Accepted October 17, 2013; Published October 19 2013

Citation: Garde K, Aravamudhan S (2013) Do Engineered Nanoparticles Penetrate into Cells? J Nanomed Nanotechnol 4: e132. doi:10.4172/2157-7439.1000e132

Copyright: @ 2013 Garde K, et al. This is an open-access article distributed under the terms of the Creative Commons Attribution License, which permits unrestricted use, distribution, and reproduction in any medium, provided the original author and source are credited. 
Citation: Garde K, Aravamudhan S (2013) Do Engineered Nanoparticles Penetrate into Cells? J Nanomed Nanotechnol 4: e132. doi:10.4172/2157$7439.1000 \mathrm{e} 132$

2. Rosi NL, Giljohann DA, Thaxton CS, Lytton-Jean AK, Han MS, et al. (2006) Oligonucleotide-modified gold nanoparticles for intracellular gene regulation. Science 312: 1027-1030

3. Howell M, Wang C, Mahmoud A, Hellermann G, Mohapatra SS, et al. (2013) Dual-function theranostic nanoparticles for drug delivery and medical imaging contrast: perspectives and challenges for use in lung diseases. Drug Deliv. and Transl. Res 3: 1-12.

4. Dykman L, Khlebtsov N (2012) Gold nanoparticles in biomedical applications: recent advances and perspectives. Chemical Society reviews 41: 2256-2282.

5. Chen J, Hessler JA, Putchakayala K, Panama BK, Khan DP, et al. (2009) Cationic nanoparticles induce nanoscale disruption in living cell plasma membranes. The journal of physical chemistry. B 113: 11179-11185.

6. Verma A, Uzun O, Hu Y, Hu Y, Han HS, et al. 2008. Surface-structure-regulated cell-membrane penetration by monolayer-protected nanoparticles. Nature materials 7: 588-595

7. Jewell CM, Jung JM, Atukorale PU, Carney RP, Stellacci F, et al. (2011) Oligonucleotide delivery by cell-penetrating "striped" nanoparticles. Angewandte Chemie 50: 12312-12315.

8. Alkilany AM, Murphy CJ (2010) Toxicity and cellular uptake of gold nanoparticles: what we have learned so far? J Nanopart Res 12: 2313-2333.

9. Van Lehn RC, Atukorale PU, Carney RP, Yang YS, Stellacci F, et al. (2013) Effect of particle diameter and surface composition on the spontaneous fusion of monolayer-protected gold nanoparticles with lipid bilayers. Nano letters 13: 4060-4067.
10. Chithrani BD, Chan WC (2007) Elucidating the mechanism of cellular uptake and removal of protein-coated gold nanoparticles of different sizes and shapes. Nano letters 7: 1542-1550.

11. Marquis BJ, Love SA, Braun KL, Haynes CL (2009) Analytical methods to assess nanoparticle toxicity. The Analyst 134: 425-439.

12. Conner SD, Schmid SL (2003) Regulated portals of entry into the cell. Nature 422: $37-44$.

13. Patra HK, Banerjee S, Chaudhuri U, Lahiri P, Dasgupta AK (2007) Cell selective response to gold nanoparticles. Nanomedicine 3: 111-119.

14. Ryan JA, Overton KW, Speight ME, Oldenburg CN, Loo L, et al. (2007) Cellula Uptake of Gold Nanoparticles Passivated with BSA SV40 Large T Antigen Conjugates. Anal. Chem 79: 9150-9159.

15. Yen HJ, Hsu SH, Tsai CL (2009) Cytotoxicity and immunological response of gold and silver nanoparticles of different sizes. Small 5: 1553-1561.

16. Hauck TS, Ghazani AA, Chan WC (2008) Assessing the effect of surface chemistry on gold nanorod uptake, toxicity, and gene expression in mammalian cells. Small 4: 153-159.

17. Cho EC, Liu Y, Xia Y (2010) A simple spectroscopic method for differentiating cellular uptakes of gold nanospheres and nanorods from their mixtures. Angew Chem Int Ed Engl 49: 1976-1980.

18. De Jong WH, Hagens WI, Krystek P, Burger MC, Sips AJ, et al. (2008) Particle size-dependent organ distribution of gold nanoparticles after intravenous administration. Biomaterials 29: 1912-1919. 